CuPAUAM. 18-1991, 261-274

\title{
APLIQUES ORNAMENTALES DE LA CABALLERIA ROMANA
}

\author{
Maria Angeles Alonso \\ SANCHEZ \\ UNIVERSIDAD AUTONOMA DE MADRID
}

\section{Resumen}

Se presentan cuatro piezas de bronce inéditas que la $\mathrm{A}$. identifica como apliques relacionados con el ejército Romano. Los tres primeros, muy semejantes entre sí, serian adornos de arnés de la caballerfa romana, datables en los siglos II-I a.C. El cuarto, con cabeza de Medusa, es un modelo muy repetido, fechable en los siglos I-III d.C.

\section{Summary}

The A display four unpublished bronze pieces, recognized as equipment related to the roman Army. The first three elements, very much alike, are harness ornaments from the roman cavalry that can be dated on the II-I c.B. C. The fourth one, a Medusa's head, is a very common patern from the I-III c.A. D.

Son numerosas y variadas las piezas metálicas que están vinculadas a las centurias de caballetía de los ejércitos romanos, tanto en relación a los jinetes como en relación a los caballos.

Entre este conjunto de piezas, nos son bien conocidas, dentro de la arqueología hispana, las que tienen una determinada funcionalidad : bocados de caballo, camas de freno, ruedas caladas, los llamados "pasarriendas"...etc, si bien los conocidos y estudiados, se encuadran, en casi su totalidad, en el periodo tardorromano. Las diversas aportaciones de Palol sobre este tema (1952, 1953, 1967, 1968, 1972, 1990) y últimamente, la síntesis de A. Fuentes (1990), dan buena cuenta de ello.

Menos conocidas son las piezas meramente ornamentales, es decir, las "phaleras", colgantes y apliques, de los que, para el periodo republicano y altoimperial, apenas tenemos noticias, probablemente porque no han sido identificados y por tanto han pasado desapercibidos en los hallazgos de las excavaciones.

Es precisamente en esas piezas en las que deseamos centrar nuestro estudio, que tiene como punto de partida la presentación de cuatro apliques metálicos inéditos que, a nuestro parecer, se encuadran dentro de lo que fue el equipamiento de la caballería romana. 
Estas cuatro piezas tienen distintas procedencias, aunque todas provienen de la zona centrooccidental de la Península, y tienen en común su carácter ornamental. Tres de ellas son muy semejantes, mientras que la cuarta es completamente distinta.

He aquí las piezas:

1. Se trata de un aplique en bronce formado por seis prótomos de animal (¿caballo?), consistentes en la cabecita, de perfil, y el cuello, que se disponen en posición afrontada dos a dos, dejando entre cada par un vacio ultrasemicircular (Fig. 1, 1, lám. I, 1). Sobte la pieza se sitúan diez bolitas aplastadas de las que seis están colocadas sobre las respectivas cabezas, otras tres en el punto de unión de cada dos prótomos, y la restante en el centro de la pieza. En el anverso se observa el pequeno saliente perforado que serviría para sujetar el aplique, probablemente a un material de cuero. La pieza mide en sus dimensiones máximas $43 \mathrm{~mm}$ por $45 \mathrm{~mm}$ y su conservación es bastante buena, aunque tiene rota la parte delantera de una cabecita y la anilla posterior de sujección.

Esta pieza fue hallada en la IV Campaña de Excavaciones realizada en el yacimiento de Castrejón de Capote (Higuera la Real, Badajoz) (1), como una más de las numerosas piezas de bronce y cerámica que se encontraban en la habitación LLE-F de un gran edificio, cuyo destino parece haber sido servir de santuario. Juntamente se encontraron dos agujas de bronce, quizá usadas para el pelo, un objeto laminar del mismo metal, consistente en diversas placas unidas entre sí por medio de remaches, otro objeto de dificil interpretación a causa del mal estado de conservación, una fibula de La Tène I, tipo Transmontano de Schüle, y dos cuentas de pasta vitrea azul, una de ellas mayor, con círculos concéntricos en amarillo. Todos estos objetos aparecieron dentro de una gran vasija de almacén, posiblemente hecha a mano, y decorada con un gran cordón con incisiones en espigado. La vasija se encontraba boca abajo, partida por la mitad intencionadamente, de suerte que sólo quedaba la boca y el cuerpo hasta la primera mitad superior, y estaba repleta de ottas piezas cerámicas (platos grises a torno, copas y cuencos de perfil en "S" hechos a mano y decorados con mamelones e incisiones).

Todo el conjunto, que ocupaba casi por completo la pequefia habitación, puede fecharse en el nivel de ocupación 2 de este castro prertomano, que ha sido datado entre el 152 a.C. (toma de la vecina Nertóbriga por las legiones romanas) y el 100 a.C., fecha en que el poblado indígena debió abandonarse por presiones políticas y de la conquista romana. No obstante los materiales podrían ser, en algunos casos, más antiguos, procedentes de las ofrendas que desde el siglo $\mathrm{V}$ a.C. se hicieron en el supuesto santuario.

2 . Se trata de una pieza semejante a la anterior, formada también por seis prótomos de animales, dos de los cuales han quedado unidos por el hocico al hacerse la fundición del bronce, por lo que el vacío que queda entre ellos es un círculo totalmente cerrado, en vez de los huecos ultrasemicirculares que se dan entre las otras dos parejas de prótomos (lám. I, 2 y Fig 1, 2). La zona central de la pieza, que se produce en la conjunción de los seis prótomos, es más ancha que en la pieza anterior, y forma un triángulo de lados curvos en el que se ve, en bajorrelieve, la cabeza de un toro visto de frente, cuyos cuernos se prolongan sobre los prótomos de la zona superior del aplique. Las

(1) Todos los datos sobre el hallazgo de esta pieza y el yacimiento donde fue encontrada, me han sido facilitados por D. Luis Berrocal Rangel, Direcror de la excavación, a quien agfadezco toda esta información, así como el que me haya facilitado la pieza para su estudio. Sobre este yaciniento ver Bertocal Rangel, L. 1989: "El asentamiento "célrico" del Castrejón de Capote (Highera la Real, Badajoz)" Cuadernos de Prehiscoria y Arqueología Universidad Autónoma de Madrid, 16, 245-295. 
medidas máximas de la pieza son $42 \mathrm{~mm}$. por $38 \mathrm{~mm}$. En el reverso puede verse, completa, la anilla de sujeción.

Esta pieza procede de Seseña Quinto, provincia de Toledo y zona muy próxima al límite con la provincia de Madrid. Fue hallada en superficie, por lo que carece de contexto arqueológico (2). El estado de conservación es francamente bueno.

3 . Esta pieza debió ser casi igual que la precedente, aunque no podemos asegurarlo dado que no se conserva de ella más que una parte, algo más de la mitad. El fragmento que nos ha llegado presenta cuatro prótomos de animal (uno de ellos roto) que se disponen en posición afrontada de dos en dos, aunque en el estado actual sólo dos prótomos cumplen este requisito, pues los otros dos han perdido su pareja (Lám I, 3 y fig. 1, 3).

En el reverso se conserva, completa, la anilla de sujeción. La pieza carece también de contexto arqueológico pues fue hallada en superficie, en la zona de Arganda, en la provincia de Madrid (2). El estado de conservación de la parte conservada de la pieza es bueno.

4. Se trata de un aplique circular, en cuyo centro puede verse, en un relieve bastante alto, la cabeza de la Medusa, con la iconografía que le es propia. Aunque bastante rodada, pueden distinguirse bien los grandes ojos, la chata nariz, la boca, todo ello en un rostro humano de forma redondeada, con el mentón muy poco señalado. Los cabellos aparecen marcados formando bandas a ambos lados de la cara y sobre la frente. Aquí, y por encima de la primera fila de cabellos, se observan dos protuberancias que pueden identificarse como las características alas. No parece, en cambio, que existan los dos cuernos que vemos otras veces en figuras semejantes. La cabeza se encuadra en el centro de un disco del que se ha perdido más de la mitad (Lám. I, 4 y fig. 2, 1), concretamente la parte que corresponde al lado izquierdo y a la zona que está bajo la barbilla de la figura, por lo que no sabemos si en esta zona habría algún detalle rematando el rostro. En la parte conservada, el disco está enmarcado por dos finas líneas concéntricas en relieve. La pieza conserva un orificio de sujeción situado sobre la cabeza, a la derecha, de forma circular, que mide $3 \mathrm{~mm}$ de diámetro. Probablemente tuvo otros tres orificios con esta misma finalidad, dos de los cuales propiciaron la rotura del disco, según puede apreciarse.

La pieza total mide $40 \mathrm{~mm}$ de diámetro, siendo la medida del saliente del relieve $11 \mathrm{~mm}$. Procede también este aplique de la zona de Arganda, en la provincia de Madrid, y fue hallado en superficie casualmente (2).

\section{PARALELOS Y CRONOLOGIA}

Respecto a las tres primeras piezas que hemos presentado y que, como ha quedado evidente, guardan una gran semejanza entre sí, (la segunda y la tercera son prácticamente iguales) conocemos una cuarta pieza muy semejante, casi igual a la que nosotros hemos descrito en el número 2, procedente del Cerro de El Gollino, en Corral de Almaguer (Toledo) (3), yacimiento todavía en

(2) Agradezco al Sz. Fernández della Cigofia que me haya facilitado estas piezas (2, 3, y 4) para su extudio. Hay quie advertir que las piezas 2 y 3, prácticamente iguales, fueron halladas en zonas muy proximas entre sí, aunque corresponden a provincias distintas (Madrid y Toledo).

(3) Agzadezco al Dr. Sanros Velasco, co-director de la excavación, los datos que me ha facilitado sobre esta pieza. Eia relación al yacimienro en que fue encontrada ver SANTOS, J. A., PEREA, A. y PRADOS, I., 1990: "Primeros resulcados de las excavaciones arqueologicas en el Cerro del Gollino (Corral de Almaguer) ${ }^{n}$, Actat del Primer Congreso de Arqueologia de la Provincia de Toledo. 309-325. 
proceso de excavación y estudio. Apareció durante la campaña de excavación de 1985 , en un departamento con abundante material arqueologico, entre el que se encontraban varios vasos de paredes finas y dos vasos de campaniense B, de la forma 5 de Lamboglia, serie 2250 de Morel, fechables entre la segunda mitad del siglo II a.C. y principios del s.I a.C.

La pieza, a la que falta uno de los prótomos del animal, está expuesta en el Museo Provincial de Toledo, y allí pudimos verla antes de ponernos en relación con el Sr. Santos Velasco, y tomar nota del yacimiento del que procedía, a la vez que constatábamos la gran semejanza que presentaba con nuestras piezas.

La cronología que da a la pieza el contexto arqueológico en el que fue hallada, coincide con la que presenta la pieza descrita por nosotros en el número 1, también con contexto arqueológico. Creemos, por tanto que todas estas piezas pueden ser datadas a lo largo del siglo II a.C. y quizá también durante el I. El hecho de que se hayan encontrado estos dos ejemplares en conjuntos cerrados, excavados con todas las garantías, es sumamente importante, pues el aspecto y la iconografia que presentan podrían inducir a errot, por la semejanza que guardan con los bronces tardorromanos o visigodos (4).

No hemos encontrado piezas iguales entre los apliques recogidos dos en las publicaciones que hacen referencia a los adornos de los arneses de las caballerías romanas, aunque si piezas parangonables, sobre todo entre los apliques compendiados por C. Boube-Piccot (1980, Fig.19-20 y 25; Lám. 51, 52, 93, 97, y 98). Son muchas y variadas las formas que estos apliques presentan, todos ellos con una finalidad meramente decorativa, provistos en el reverso de la anilla de sujeción y con tamaños que varían entre 30 y 100 mm como medidas máximas. Aunque para muchos de estos bronces de Marruecos carecemos de la información suficiente en cuanto al contexto arqueológico en el que han sido halladas, sin embargo Boube-Piccot las asocia, al menos en una gran parte, a la presencia de las centurias de caballería en la provincia durante los siglos I y II d. C., en destacamentos, a veces incluso de veteranos, instalados en Volúbilis, Tocolosida, Ain Chkour, Tanger, Banasa, Sala ...etc.

También tenemos testimonios literarios y arqueológicos de adornos metálicos en los arneses de caballo de los íberos, al menos en la Baja Epoca. Emeterio Cuadrado (1948, 279-280, fig. 3, 7) en su estudio sobre los arreos ecuestres de los exvotos con figuras equinas, procedentes del Santuario del Cigarralejo, habla de los adornos metálicos de los que afirma que "estarían calados o grabados y hasta tal vez niquelados de cobre o plata".

En cuanto a la cuarta pieza, es decir, al aplique circular con cabeza de Medusa, tenemos en Espafia un claro paralelo en la "phalera" romana de Encinas de Abajo (Salamanca), encontrada casualmente mientras se realizaban labores de labranza, y publicada en la Revista de Arqueologia de octubre de 1989 (L. Arias González y otros, 1989, 46-47). Nuestra pieza, sin embargo, se diferencia de la de Encinas de Abajo en varios detalles:en primer lugar en el tamaño, pues su diámerro es de sólo 40 mm, y también en su elaboración, que es menos cuidada. Además el estado de conservación es mucho peor: ha perdido más de la mitad del disco que rodea la cabeza, y está tan rodada que no es posible apreciar si tiene señalado o no el iris de los ojos. Incluso lo que pudieron ser cuernos está tan desgastado que es arriesgado afirmar que los tuvo. No es posible tampoco saber si tuvo un remate por debajo de la barbilla, y en caso afirmativo cómo era, pues falta esa zona del disco.

(4) Cfr. WERNER, J, 1935, Munedatietre Austrasische Grabfunde, lám 25, 5 y 27, 2. WOLFANG, H, 1974: Germanische Grabfunden, Lams, 106, 5 a; 115,8 y 10; y 120, 5 . 
Dado que nuestra pieza (y también la de Encinas de Abajo) es fruto de un hallazgo casual, sin ningún contexto arqueológico, no es posible asignarle una datación precisa. Piezas semejantes se fechan en los siglos I-III. (5)

Estos discos o fáleras, mostrando la cabeza de la Medusa, son relativamente frecuentes en el ámbito romano, también en provincias, y su vinculación al mundo militar es evidente. No siempre fueron recompensas militares concedidas a las centurias de caballería. También se usaron como adornos de los carros. De Volubilis procede un aplique con representación de la Medusa vinculado a un carro (Boube-Piccot 1980, Lám. 19, número 41 del inventario) y otros seis apliques con el mismo tema han sido hallados con el carro de Salónica (G. Seure 1904, 218, fig. 16). Ignoramos por tanto cuál sería su verdadera función.

\section{FALERAS Y APLIQUES EN LAS FUENTES ESCRITAS Y EN LA DOCUMENTACION ARQUEOLOGICA}

El término "phalera", que en latín aparece siempre en plural es de origen griego y viene a indicar "algo brillante". Los romanos lo usaron para significar cosas en sí distintas, aunque semejantes, en cuanto que se trataba de objetos ornamentales, metálicos, relacionados con el ejército. En primer lugar se usó este término para denominar unos discos metálicos, frecuentemente con relieves de divinidades o de bustos de emperadores, que se concedían como recompensas militares. Su adjudicación y uso sufrió, a lo largo del tiempo, una evolución que nos ha llegado a través de las fuentes escritas y de las representaciones iconográficas. Se empleó también este término para significar unos discos metálicos, frecuentemente adornados, al menos con incisiones concéntricas, cuyo papel fundamental, además del ornamental, era servir para unir las correas de los arneses de los caballos. Estas fáleras suelen tener en su reverso unas anillas (generalmente tres, aunque también pueden ser dos o cuatro), a las que se sujetarían dichas correas, o una especie de estribos laterales que tenían el mismo fin. (Hay que advertir que a veces se ha dado el nombre de "fáleras" a unas piezas que más bien deben ser identificadas como camas de frenos). Pero creemos que el término fálera se usó también para designar los adornos que se sujetaban a las correas de los arneses de los caballos, principalmente a las bridas, sin ninguna función utilitaria, puesto que en las fuentes escritas no aparece otro término que el de fálera (suele decirse "equus phaletatus") y sin embargo, tanto la iconograffa de los caballos como los hallazgos arqueológicos han proporcionado, además de los consabidos discos para unir las correas, otros adornos y apliques cuyo objetivo es meramente ornamental. También aparecen estos discos ornamentales y apliques variados, en la ornamentación de los carros. Se trata, pues, de un término polivalente, que se refiere a objetos de finalidades diversas.

Son muchos los textos antiguos que hablan de las fáleras, tanto en su papel de distinciones militares como en su función de elementos de unión o de decoración de caballos y carros.

Homero habla ya de un casco de oro decorado con cuatro fáleras, y del casco de Ayax, enjoyado con estos mismos adornos. También Esquilo, al describir la tiara de Darío, se refiere a la fálera que la adornaba (es rara la existencia de una fálera única). Fáleras sujetando o adornando los arne-

(S) De fecha ciertamente unás temprana y con notables diferencias de realización, aunque cambién con el cema de la Medussa, son dos apliques expuestos er el Museo Numantino de Soria y publicados recientemence en el B.S.A.A.V. (García Merino 1990 , 244-247). Según este eseudio se trata de piezas pertenecientes al ajıar y ornamentación domésticos, fechables en la erapa julio-claudia, que nada tienen que ver con las falleras o apliques relacionadios con la cabalieria romana, aunque coincida el remsa. 
ses de los caballos pueden verse en los relieves del altar de Pérgamo, en el mosaico pompeyano de Alejandro, en los vasos pintados griegos... Herodoto alude a la riqueza de los Masagetes quienes adornaban sus caballos con fáleras de oro (Dognée, 1867).

Fueron los etruscos quienes aportaron a estos ornamentos una mayor importancia y quienes según Festo- transmitieton su uso a los romanos. Alföldi $(1979,17-25)$ ha reunido en una interesante publicación todo un conjunto de textos griegos y latinos en los que se habla tanto de estos distintivos milirares como de las fáleras de uso ornamental (6). En cuanto a los primeros, los textos documentan que, en un principio, tales distintivos fueron signo de nobleza. Dionisio de Halicarnaso, al hablar del recibimiento dispensado a Cincinatus, nombrado "magister populi", alude a la presencia de caballos adornados con fáleras y, aunque el relato de tal recibimiento es, a juicio de Alföldi, inventado, sin embargo no deja de ser un documento fidedigno en cuanto al uso de las fáleras como insignias que indicaban una distinción. También Tito Livio nombra un "equus phaleratus" en relación a los tristes presagios acaecidos sobre uno de los dos cónsules del anio 43 a.C. y A. Rein (1860, 186 A.2) confirma que en este caso sólo podía tratarse del adorno que indicaba el rango de los caballos consulares. Efectivamente, Tácito y otros autores nos documentan la existencia de esta distinción para los caballos consulares durante el Imperio. Sin embargo parece que no sólo se concedieron fáleras a los caballos consulares, sino que ya a finales de la república le correspondía un "equus phaleratus" también a los dignatarios inferiores. Las investigaciones de Alföldi han individualizado a estos dignatarios inferiores en la clase social que se colocaba entre el orden senatorial y la plebe, es decir, la clase equestre, de origen más reciente respecto a la antigua nobleza romana, pero de sólida fortuna económica. Precisamente los pertenecientes a esta clase social estaban en los municipios periféricos y en las colonias itálicas y provinciales, lo que avala la tesis de que estas piezas aparezcan en los yacimientos hispanos.

Cicerón relata en una de sus cartas que C. Munatius Plancus, designado pretor por César para el año 43, pero que antes había organizado como triunviro la colonia de veteranos de Bathroton, en el Epiro, fue expulsado de allí con todas sus gentes y para podex escapar más fácilmente, hizo quitar las insignias de su dignidad, es decir escapó "sine phaleris".

Testimonio de esta dignidad representada por las fáleras es el hecho de que el senado acostumbraba a regalar a los reyes extranjeros "equos phaleratos". Tito Livio alude al regalo hecho a Massinissa de "equos duos phaleratos", además de un vestido rojo, fibulas de oro y otros presentes.

Otro testimonio directo de las fáleras que enriquecían los caballos de los generales nos lo da el relato de Plutarco sobre cómo pudo escapar con vida Pompeyo, vencido momentáneamente por Sertorio. Parece ser que, al verse en peligro, entregó a sus enemigos su caballo adornado con fáleras, $y$ asi pudo escapar de la muerte.

Que las fáleras, tanto las que se concedían como insignias militares personales como las que adornaban los caballos, eran (por lo menos al principio) sefial de rango y propias de la clase aristocrática nos lo prueba el hecho que relata Tito Livio de que, cuando en el año 304 a.C. la asamblea popular eligió "aedilis curulis" a Cn. Flavio, que era hijo de un liberto, se indignó tanto la nobleza que, como señal de protesta, sus componentes abandonaron las insignias de su rango, "plerique nobilium anulos aureos et phaleras deponerent". El mismo hecho es relarado por Plinio.

Un texto de Virgilio documenta, a propósito del entierro de Pallas, la costumbre de despojar a los caballos de los discos ornamentales, como una manifestación de duelo.

(6) Nos remitimos a la publicación de este autor para todas las referencias bibliograficas de los autores gziegos y latinos. 
Respecto al hecho de que las fáleras fueron un atributo propio de las centurias patricias de caballeria, nos lo confirma el suceso narrado por T. Livio de que, cuando en el anio 216 a.C. murió en el campo de batalla de Cannas una gran parte de la juventud patricia de Roma, Aníbal se adueñó de los discos de plata de sus caballos, y especifica que era mucha la plata que había en las "phaleris equorum". Sin embargo, como ya hemos indicado, con el pasar del tiempo y con los cambios producidos en las estructuras de la sociedad romana, las centurias de caballería perdieron su exclusivismo aristocrático y, lógicamente, las fáleras fueron perdiendo su papel indicativo del orden social, asumiendo la mera función de condecoraciones militares, cuyo uso se extendió de modo notable a las provincias.

Los testimonios arqueológicos de las fáleras nos han llegado a través de la iconografia de los caballeros romanos y sus caballos, y también a través de los hallazgos arqueológicos. (Aquí nos concretaremos a las fáleras que aparecen en los arneses de los caballos, pues son las que nos interesan en relación a las piezas inéditas que hemos presentado).

Dos publicaciones recientes han recogido una amplia documentación sobre el tema, y a sus aportaciones vamos a referirnos. En primer lugar el estudio de M. C. Bishop (1988) titulado "Cavalty Equipment of the Roman Army in the first century A. D.". Las fuentes de información utilizadas por este autor han sido, de una parte, los monumentos funerarios de los jinetes romanos, tan abundantes en los museos centroeuropeos, en los que tantas veces aparece en relieve el caballero difunto montando su caballería de combate, o bien el difunto participando del banquete funerario, mientras que en la parte inferior del monumento se ve su caballo equipado con todos sus arneses. Junto a estos monumentos funerarios, que constituyen lo que podríamos llamar la escultura privada, está la escultura oficial, cuyos relieves históricos son un importante elemento informativo sobre el ejérciro romano. El autor ha recogido los datos proporcionados por la columna trajana, por los relieves de los frisos del arco triunfal de Orange, por los del mausoleo de SaintRemy-de-Provence, y por los del Trofeo trajaneo de Adamklissi. También tiene en cuenta las aportaciones de las pequeñas figuras de caballos hechas en cerámica, y las representaciones de los mismos sobre algunas piezas metálicas, como son el caldero de Gundestrup (sin duda antetior al s.I a.C.) y uno de los yelmos de Nawa, ciertamente posterior. En todos estos monumentos y piezas aparecen representaciones de caballos que llevan fáleras, bien como piezas de unión, bien como elemento del que pende un colgante o como pieza de adorno de las bridas y correas del arnés. El estudio pormenorizado de todas las piezas metálicas que aparecen en los arneses de los caballos, Lleva al autor a la conclusión de que los romanos adoptaron el equipo de la caballería celta, puesto que las partes funcionales del arnés no cambian significativamente desde los tiempos del caldero de Gundestrup (siglos III-II a.C.) hasta las que aparecen en la representación de la tumba de T. Flavius Bassus, de época flavia (Fig. 2, 2 y Fig. 3).

La segunda publicación a la que deseamos referimos es el extenso estudio de C. Boube-Piccot (1980) sobre Les bronzes antiques du Maroc, y concretamente el volumen III titulado Les Chars et l'attelage. Aquí la autora ha inventariado y clasificado un conjunto de 630 piezas provenientes de Volubilis, Tocolosida, Banasa, Souk-el Arba del Rharb, Thamusida, Sala, Tamuda, Lixus y Tanger, depositadas en el momento actual en los Museos de Rabat, Tetuán y Tanger. Dentro del conjunto de piezas clasificadas como elementos de arnés (existen otras piezas vinculadas exclusivamente a los carros), distingue la autora tres tipos: en primer lugar los bocados de caballo, con sus piezas laterales. En segundo, los elementos de unión y fijación de las correas. Y en tercero, los elementos exclusivamente decorativos. Es entre estos elementos ornamentales donde hemos encontrado los paralelos más claros para las tres primeras piezas, hasta ahora inéditas, que presentamos en este tra- 
bajo, y concretamente con las que la autora incluye dentro del apartado "apliques" (Fig. 4), como ya hicimos constar anteriormente.

\section{ICONOGRAFIA DE NUESTROS APLIQUES}

Prescindimos aquí de la fálera con cabeza de Medusa por ser un tema suficientemente identificado y conocido. Nos referiremos, por tanto, solamente a los tres primeros apliques por nosotros presentados, formados, cada uno, por seis prótomos de animales que se disponen, radialmenre, afrontados de dos en dos. Uno de los apliques presenta la particularidad, como ya dijimos, de tener en bajo relieve, una cabeza de toro, vista frontalmente, en la parte central de la pieza Y otro está adornado con seis bolitas achatadas dispuestas en distintos puntos del aplique.

Tenemos pues, como temas iconográficos principales, las cabezas de animales. Aunque elaborados rudamente y sin detalles, nos parece poder identificar en los prótomos de animales, pequenas cabezas de caballo. La cabeza de toro, vista frontalmente, no ofrece duda.

Tanto las representaciones de caballos como las de toros son corrientes en la iconografia pretromana y tomana hispánica. Respecto al caballo, las fuentes escritas y también la documentación arqueológica evidencian su importancia para la economía y las actividades guerreras, justificando, a juicio de Blázquez (1983, 101), la existencia de una diosa protectora de caballos o un despotes. Las numerosas placas con relieves de caballos encontradas en el Cigarralejo y en Pinos Puente (ambos yacimientos en la provincia de Murcia) atestiguan estos cultos en el área ibérica. Pero también se atestiguan sus representaciones en las zonas lusitanas y carpetanas, e incluso en otras áreas. Las fuente literarias aluden a la consideración del caballo como animal sagtado entre los pueblos del norte.

El rema de los prótomos de caballos en Hispania es relativamente frecuente en los adornos de arneses, ya desde los primeros siglos de la República y pervive hasta época visigoda. Contamos con claros testimonios, como son los bocados de caballo, cuyas placas laterales presentan prótomos equinos, encontrados en el ala oriental del edificio amortizado de Cancho Ruano, en Zalamea la Serena (Badajoz), cuyos materiales se han fechado entre los años 430 y 370 a.C. (Maluquer de Motes, Aubet, 1981). También la cama de bocado de caballo publicada por Blázquez (1984) procedente de Cástulo, y la serie de piezas tardorromanas dadas a conocer en su mayoria por Palol y que, en casi su totalidad, son ruedas caladas o camas de frenos (Palol 1952, 1953, 1960, 1967, 1972 y 1990).

Con respecto al toro, es algo comunmente aceptado que se trate de un culto de origen preindoeuropeo, que aflora después, perviviendo en épocas muy posteriores. Según Blázquez (1957 y 1983) el área de difusión de este culto al toro se extiende desde la zona media del Ebro hasta el macizo montañoso iberico, siendo los focos principales la zona del Moncayo (Soria y parte de Cuenca), la región montafiosa de Gredos y las provincias de Avila, Cáceres y Toledo, dentro del sistema oretano. Este culto ha dejado su testimonio en las representaciones de toros en piezas de bulto redondo y también en las figuras que aparecen en las monedas, tanto del ciclo griego como del ciclo romano. Según Blázquez, el toro como símbolo de fuerza fecundadora "va unido a las creencias astrales de inmortalidad" .

A. Serrano $(1957,106)$ ha sefialado para la época prerromana relaciones frecuentes entre los pueblos de la meseta y los del sur y sureste. Fruto de esas relaciones serían las corrientes de influencia mutua que es posible rastrear en orden a la escultura zoomorfa, que se desarrollarfa "primero en el S y S.E. debido a las intensas influencias de pueblos mediterráneos, y tal vez en el siglo III, 
cuando las relaciones con los pueblos de la Meseta se intensifican, es precisamente cuando esta escultura zoomorfa de la zona ibérica influye en el rudimentario arte celta y surgen estas toscas y enigmáticas figuras de toros y cerdos".

Nuestras piezas son una prueba más de esa corriente animalística que llega y se desarrolla con fortuna en las zonas lusitana y carpetana. 


\section{BIBLIOGRAFIA}

ALFOLDI, A., 1979 : Der Frïhrömische Reiteradel und seine Ehrenabzeichem, Roma, 17-25.

ARIAS GONZAiez, Z. y OTROS, 1989 : "La phalera romana de Encinas de Abajo", Revista de Arqueologta, I02, $46-47$.

Berrocal Rangel, Ln, 1989: "El asentamiento céltico del Castrejón de Capote (Higuera la Real, Badajoz)", Cuadernos de prehistoria y Arqueologia Universidad Autonoma de Madrid, 16, 245-295.

BISHOP, M. C., 1988: "Cavalry Equipment of the Roman Army in the first century A.D. ", Military Equipment and the Identity of Raman Soldiers, (Coulston ed.), 67-195.

BlazQuez MaRTineZ, J. M., 1983: Primitivas religiones ibericas. II Religiones Prerromanas. Madrid.

1.. 1984 Castulo IV. E.A.E., 41-51.

Boube-PiCCOT, C., 1964: "Une Phalère de harnais a decor de trompetes", BAMaroc 5, 183-193.

... 1980: Les bronzes antigues du Maroc. III Les chars et l'attelage. Erudes et Travaux d' Archeologie Marocaine, 8.

CuADRADO, E., 1948: "Arreos de montar ibericos de los exvotos del santuario del Cigarralejo", Crónica del IV Congreso Arqueologico del Sudeste Español, Elche, 279-280.

DOGNEE, E., 1867: Les phaleres des guerriers romains, Caen, 9 y ss.

FUENTES, A., 1990: "Los bronces bajoimperiales en Hispania", Los bronces romanos en España (Catálogo de la Exposición), Madrid, 119-122.

MAiUQUER DE MOTES, J; AUBET, M. E., 1981: Andalucta y Extremadura. IV "El santuario protohistórico de Zalamea la Serena (Badajoz)" Barcelona, 225-409.

Palol, P., 1952: "Algunas piezas de adorno de arnés de época tardorromana e hispanovisigoda, Archivo Español de Arqueología, 25.

... 1953-54: "Bronces de arnés con representaciones zoonóficas" Ampurias 15-16.

... 1960: "Dos piezas de arnés con representaciones de caballos", Oretania 5.

... 1972: "Una tumba romana de Toledo y los frenos de caballo hispanorromanos del Bajo imperio", Pyrenae 8.

.. 1990: "Bronces cristianos de época romana y visigótica", Los Bronces Romanos en España (Catálogo de exposición). Madrid, 137-139.

REin, A., 1860: Annali dell' Istituto, 186 A.2.

SANTOS, J. A. y OTROS, 1990: "Primeros resultados de las excavaciones arqueológicas en el Cerro del Gollino (Corral de Almaguer)", Actas del Primer Congreso de Arqueologia de La Provincia de Toledo, Toledo 309. 325.

SERRANO, A., 1957: "Observaciones sobre la distribución geográfica de la escultura zoomorfa prerromana", Zephyrus VIII, Salamanca 103-110.

SEURE, G., 1904: B. C. H., 218, $\mathbf{n}^{\circ} 17$, fig. 16. 

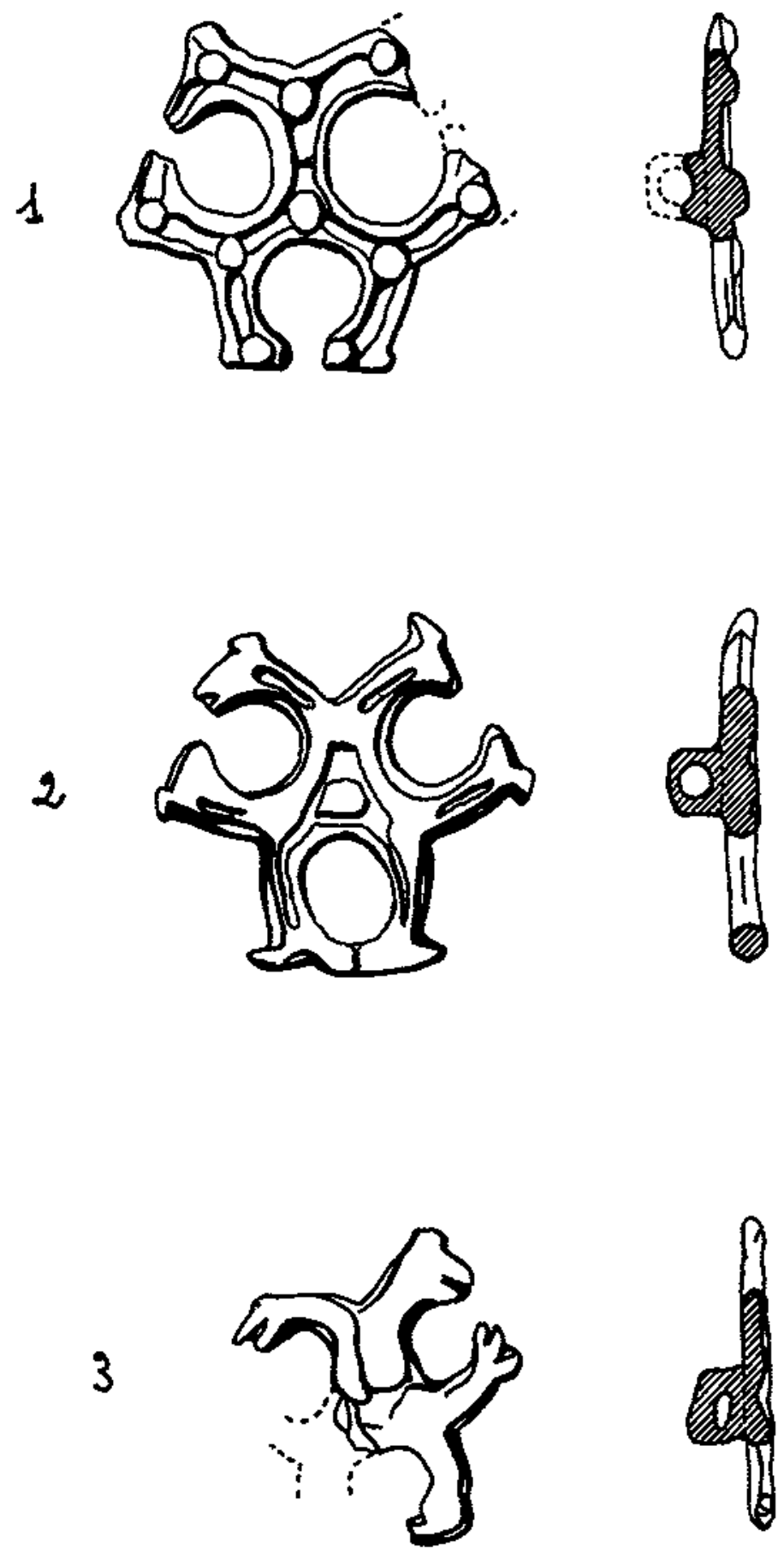

Fig. 1: 1, 2 y 3: apliques con prótomos de animales. 

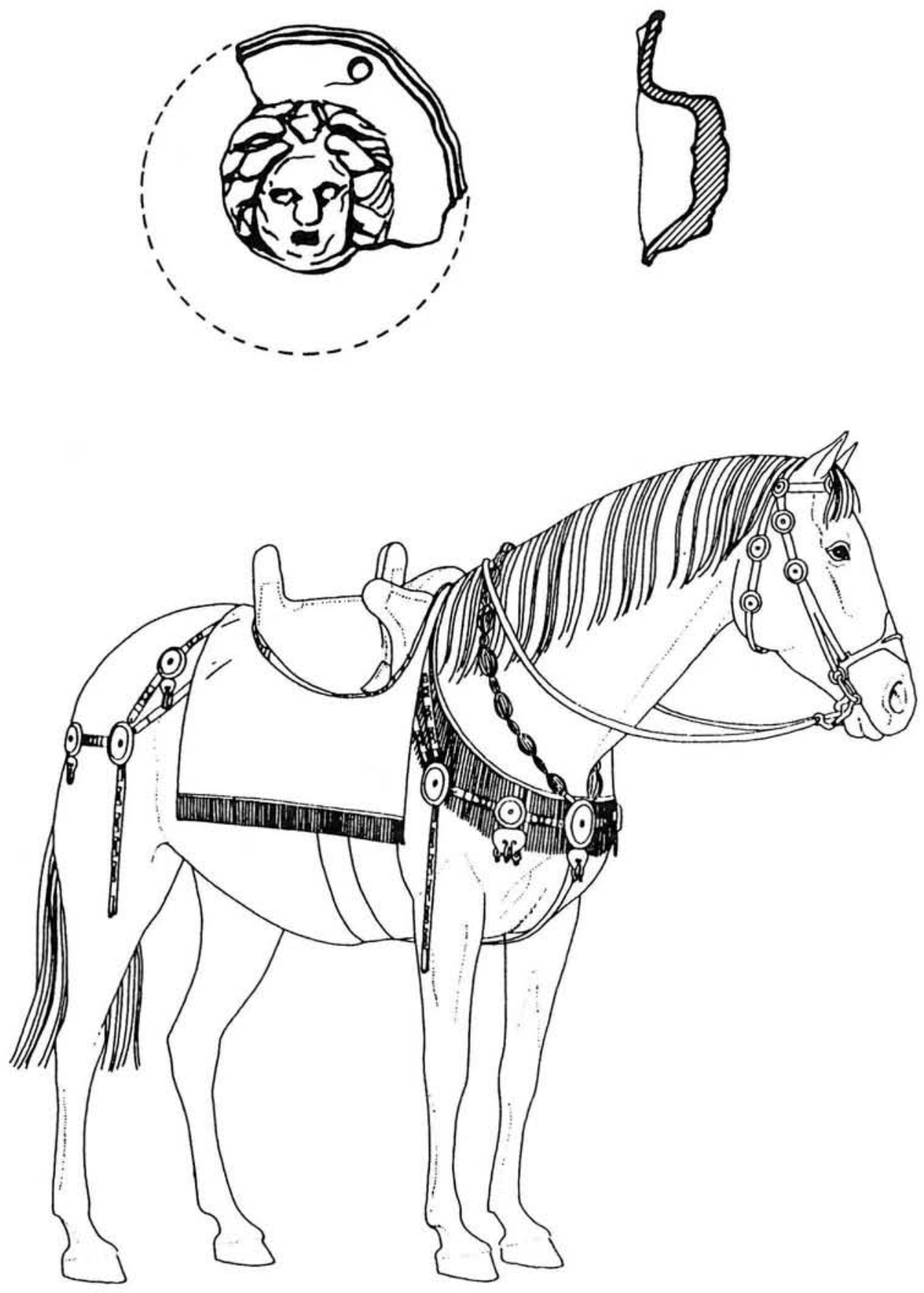

Fig. 2: 1 aplique con cabeza de Medusa; 2 reconstrucción del arnés romano de época flavia (según Bishop). 


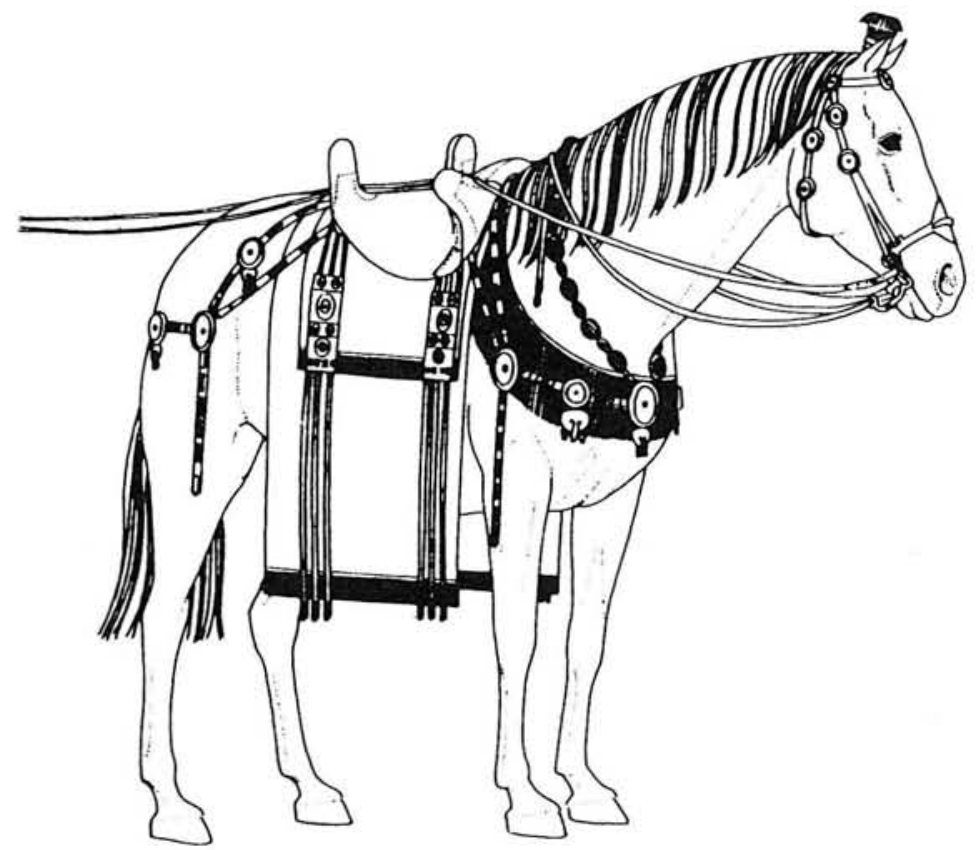

Fig. 3: reconstrucción del arnés de desfile típico de época flavia, con bridas largas (según Bishop).

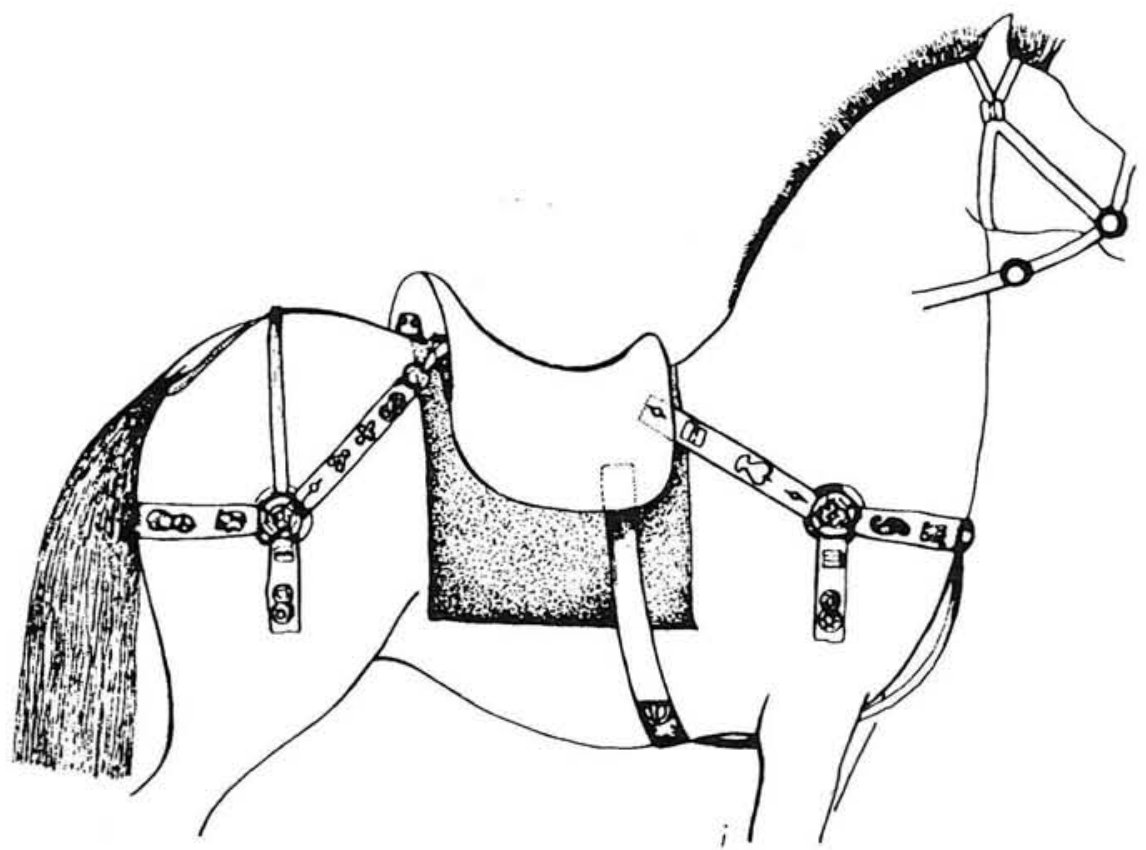

Fig. 4: reconstrucción de los arneses descubiertos en dos tumbas de caballos de Brigetio (según Barkoczi). 

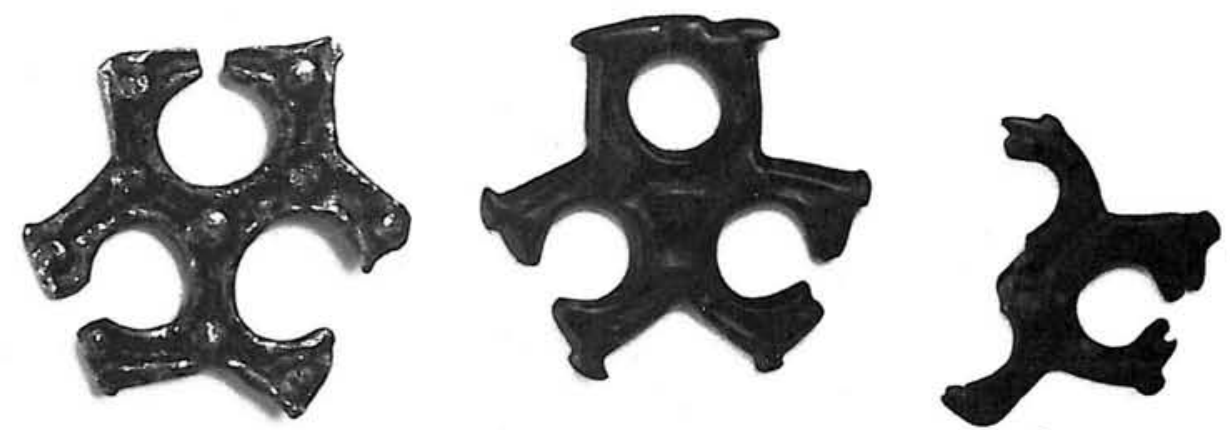

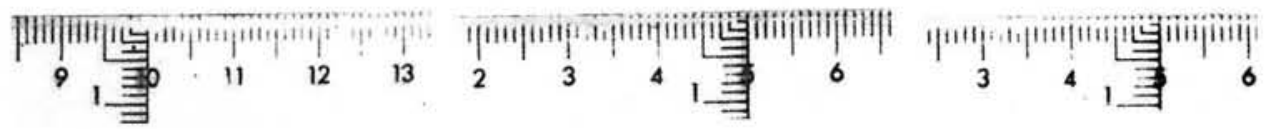
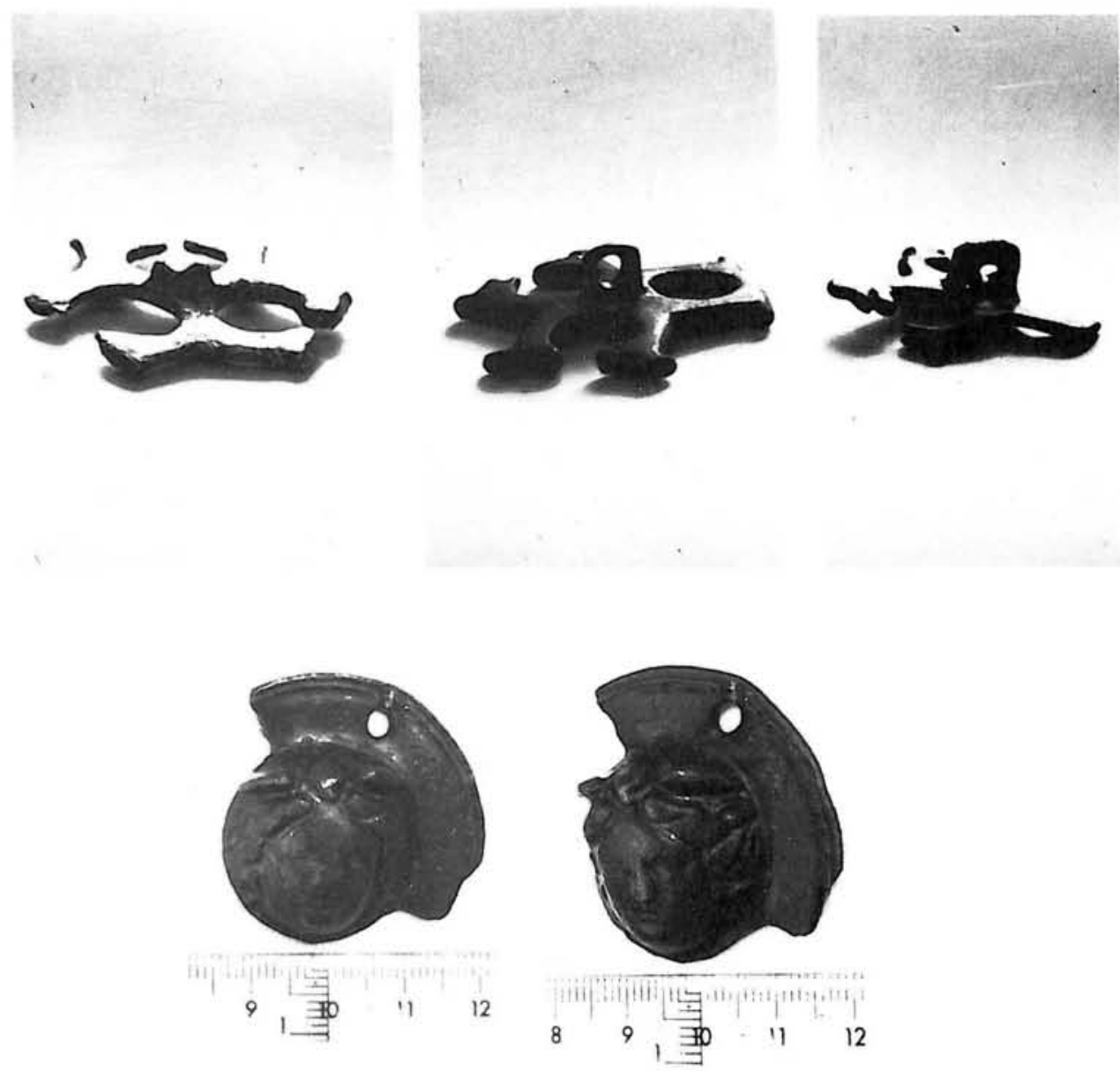

Lám. I: 1, 2 y 3: apliques con prótomos de animales, anverso y reverso. 4: aplique con cabeza de Medusa. 